\title{
Long-Term Cultured Hairy Roots of Chicory-A Rich Source of Hydroxycinnamates and 8-Deoxylactucin Glucoside
}

\author{
Janusz Malarz • Anna Stojakowska • Wanda Kisiel
}

Received: 17 June 2013 / Accepted: 12 August 2013 /

Published online: 25 August 2013

(C) The Author(s) 2013. This article is published with open access at Springerlink.com

\begin{abstract}
A 12-year-old hairy root culture of Cichorium intybus L., a callus culture of the plant as well as roots and leaves of a wild plant of chicory, and roots of two C. intybus L. var. sativum cultivars were examined in respect of their hydroxycinnamate and sesquiterpene lactone compositions and contents. Total phenolics and diphenylpicrylhydrazyl radical scavenging activity of the examined plant tissues were also analyzed. The most active in radical scavenging were extracts from the hairy roots and leaves of chicory. 3,5-Dicaffeoylquinic acid was the major antioxidant present in the hairy roots. Its content in the root biomass reached $5.5 \%$, calculated on a dry weight basis. 8-Deoxylactucin glucoside (crepidiaside A) was the major sesquiterpene lactone in the hairy roots. Its content reached $1.4 \%$, calculated on a dry weight basis, and was nearly two orders of magnitude higher than that in the roots of wild chicory plant. The glucosidic derivative of 8-deoxylactucin constituted over $85 \%$ of the total sesquiterpene lactone content in the long-term cultured hairy roots of chicory. Aglycone of this compound was reported to possess anti-inflammatory activity. The qualitative and quantitative analyses of hydroxycinnamates in callus and hairy root cultures of $C$. intybus were undertaken for the first time.
\end{abstract}

Keywords Chicory $\cdot$ Hairy roots $\cdot$ Hydroxycinnamates $\cdot$ Antioxidants $\cdot 8$-Deoxylactucin glucoside

$\begin{array}{ll}\text { Abbreviations } & \\ \text { 5-CQA } & \text { Chlorogenic acid } \\ \text { CTA } & \text { Caftaric acid } \\ \text { DCQA } & \text { Dicaffeoylquinic acid } \\ \text { DCTA } & \text { Cichoric acid } \\ \text { DPPH } & \text { 2,2-Diphenyl-1-picrylhydrazyl } \\ \text { DW } & \text { Dry weight } \\ \text { FW } & \text { Fresh weight }\end{array}$

J. Malarz $(\bowtie) \cdot$ A. Stojakowska $\cdot$ W. Kisiel

Department of Phytochemistry, Institute of Pharmacology, Polish Academy of Sciences,

Smętna Street 12, 31-343 Kraków, Poland

e-mail: malarzj@iff-pan.krakow.pl

A. Stojakowska

e-mail: stoja@if-pan.krakow.pl

W. Kisiel

e-mail: kisielw@if-pan.krakow.pl 
RP-HPLC/DAD Reversed-phase high-performance liquid chromatography with photodiode array detection

\section{Introduction}

Chicory (Cichorium intybus L., Asteraceae, tribe Cichorieae) is a wild plant species native to Europe, Western Asia, and Northern Africa. It is known as a traditional herbal remedy used to promote appetite and digestion. Commercially grown root cultivars of the plant are raw materials for production of polyfructans $[1,2]$. The most characteristic secondary metabolites of C. intybus are bitter-tasting sesquiterpene lactones (mainly lactucin-like guaianolides) [3] and phenolics (hydroxycinnamates and coumarins) [4-7]. Lactucin-like guaianolides are potent anti-inflammatory agents. Of these, 8-deoxylactucin was reported to inhibit DNA binding of the transcription factor NF-kB and was identified as an inhibitor of cyclooxygenase-2 [8]. It also showed particularly high nitric oxide inhibitory activity [9]. Analgesic and sedative activities of lactucin-like guaianolides were confirmed by Wesołowska et al. [10]. Plant hydroxycinnamates are either free acids - hydroxyderivatives of cinnamic acid (e.g., caffeic, ferulic, synapic, and $p$ coumaric acids) or their conjugates. One of the most commonly occurring conjugates is 5caffeoylquinic acid (5-CQA) also known as chlorogenic acid. Dicaffeoylquinic acids (DCQAs) along with tri- and tetracaffeoylquinic derivatives as well as mono- and dicaffeoyl conjugates with dicarboxylic aliphatic acids, most commonly tartaric and succinic acid, are characteristic constituents of Asteraceae plants. The compounds are well-documented radical scavengers and lipid peroxidation inhibitors [11-13]. They possess skin protecting activity against UV-induced damage [14, 15] and antiviral activity [16-18]. Moreover, caffeoylquinic acids play a role as infection and insect infestation inhibiting factors in plants $[19,20]$.

Hairy root cultures may provide an alternative source for production of root-derived biologically active compounds at high and stable levels [21]. In our previous study, sesquiterpene lactones were isolated from Agrobacterium rhizogenes LBA 9402 transformed hairy roots of $C$. intybus [22]. High activity of a hydroalcoholic extract from the hairy roots in scavenging of the 2,2-diphenyl-1-picrylhydrazyl (DPPH) free radical prompted us to investigate phenolic constituents of the extract. This led to the isolation of four known hydroxycinnamates, including caffeic, 5-CQA, 3,5-DCQA, and 4,5-DCQA acids, and a new neolignan glucoside - (7S, 8R)-3'demethyl-dehydrodiconiferyl alcohol-3'-O- $\beta$-glucopyranoside [23]. In addition, the sesquiterpene lactone 8-deoxylactucin glucoside was obtained for the first time from the hairy roots of C. intybus.

The aim of the present study was to evaluate the hairy roots of chicory as a source of hydroxycinnamates and sesquiterpene lactones based on comparisons of compositions and contents of these compounds, total phenolic contents, and antiradical activities, with those of chicory of wild origin, commercial varieties of root chicory, and callus culture of the plant.

\section{Material and Methods}

\section{Standards and Reagents}

Caftaric acid (CTA, purity $>97 \%$ ) was purchased from Sigma-Aldrich Co. (St. Louis, MO, USA). Cichoric acid (DCTA, purity $>98 \%$ by HPLC), 5-CQA (purity $>97 \%$ by HPLC) and 1,3-dicaffeoylquinic acid (cynarin, purity $>99 \%$ by HPLC) were purchased from Roth (Karlsruhe, Germany). 3,5-DCQA and the sesquiterpene lactone 8-deoxylactucin glucoside (crepidiaside A) were isolated in our laboratory from Lactuca virosa and C. intybus hairy 
root cultures, respectively [23, 24]. Purities of the compounds were $\geq 90.0 \%$ (by HPLC). The remaining sesquiterpene lactones: 8-deoxylactucin, jacquinelin, jacquinelin glucoside (crepidiaside B), lactucin, 11ß,13-dihydrolactucin, and lactucopicrin were isolated in the course of our previous studies from different Cichorium and Lactuca species [3, 25-27]. $\mathrm{MeOH}, n$-hexane, and chloroform of analytical grade were purchased from POCh S.A. (Gliwice, Poland). Water was purified by a Milli-Q system (Millipore Corp., Bedford, MA, USA). $\mathrm{MeOH}$ and MeCN of HPLC grade as well as formic acid and glacial acetic acid were purchased from Merck (Darmstadt, Germany).

\section{Plant Material}

Leaves and roots of C. intybus L. and roots of C. intybus L. var. sativum (cultivars Dageraad and Sabau 3) were harvested during the first year of their vegetation period from plants grown in the glasshouse of the Garden of Medicinal Plants, Institute of Pharmacology, Polish Academy of Sciences (Kraków, Poland). A callus tissue and hairy roots of $C$. intybus were harvested from in vitro cultures maintained for over 10 years in our laboratory. The cultures were obtained and cultivated as described elsewhere $[22,28]$. The hairy root culture of $C$. intybus, transformed by $A$. rhizogenes, was established from aseptic seedlings of the plant obtained from seeds of known wild origin, delivered by the Botanical Garden of Free University in Berlin (Germany). A. rhizogenes strain LBA 9402, containing agropine type Ri plasmid, pRi 1855, was used in the experiment. The hairy roots were induced on leaf explants, excised from the seedlings, and their transformed nature was proven by opine assay and rol genes detection in the plant genomic DNA. The roots were cultivated in a modified liquid MS medium [29], containing $1 / 2$ strength macronutrients and $3 \%$ sucrose, on a gyrotory shaker $\left(110 \mathrm{rpm}\right.$.), at $25^{\circ} \mathrm{C}$, under cool white fluorescent tubes $\left(20 \mu \mathrm{E} \mathrm{m}^{-2} \mathrm{~s}^{-1}\right)$ with a 16-h photoperiod, and were subcultured every 4 weeks by inoculating $0.7 \mathrm{~g}$ of roots in 250-ml Erlenmeyer flask with $40 \mathrm{ml}$ of the fresh nutrient medium. A time course experiment was performed by harvesting roots 5, 10, 15, 20, and 28 days after the inoculation into the fresh medium. Fresh (FW) and dry (DW) weights of roots, as well as hydroxycinnamate and lactucin-like guaianolide contents were estimated in the harvested plant material. The experiment was done in triplicate.

Quantification of Hydroxycinnamates and Caffeic Acid

\section{Sample Preparation}

The dried and pulverized plant material $(0.1 \mathrm{~g})$ was extracted with $70 \% \mathrm{MeOH}(10 \mathrm{ml})$ at room temperature for $3 \mathrm{~h}$ on a rotary shaker $(100 \mathrm{rpm})$. The mixture was filtered and the residue extracted once more with $10 \mathrm{ml}$ of the fresh solvent. The extracts were combined and evaporated to dryness under reduced pressure. The dry residue was redissolved in $70 \%$ $\mathrm{MeOH}(1 \mathrm{ml})$ and centrifuged $(11,340 \times g, 5 \mathrm{~min})$ prior to HPLC analysis.

\section{RP-HPLC Analysis}

Analytical RP-HPLC separations of the samples were performed using an Agilent 1200 Series HPLC system (Agilent Technologies, USA) equipped with a Rheodyne manual sample injector, quaternary pump, degasser, column oven and a diode array detector. Chromatographic separations of hydroxycinnamates were carried out at $25^{\circ} \mathrm{C}$, on a Zorbax Eclipse XDB-C18 column, $4.6 \times 150 \mathrm{~mm}$ (Agilent Technologies, USA), with a mobile phase consisting of $\mathrm{H}_{2} \mathrm{O} / \mathrm{HCOOH} / \mathrm{CH}_{3} \mathrm{COOH}$ 99/0.9/0.1 (solvent A) and $\mathrm{MeCN} / \mathrm{MeOH} / \mathrm{HCOOH} / \mathrm{CH}_{3} \mathrm{COOH}$ 
89/10/0.9/0.1 (solvent B), at a flow rate of $1 \mathrm{ml} \mathrm{min}^{-1}$, using $5 \mu \mathrm{l}$ injections. The gradient elution conditions described by Spitaler et al. [30] were applied. The analysis allowed identification of the following hydroxycinnamates present in the plant material: CTA, Rt $-6.6 \mathrm{~min}$; 5CQA, Rt-7.3 min; caffeic acid, Rt-8.5 min; DCTA, Rt-20.3 min; 3,5-DCQA, Rt-28.2 min, and 4,5-DCQA, Rt-36.10 min (Fig. 1), based on their retention time values, online UV spectra and by co-chromatography with standard samples. The compounds were quantified using four-point calibration curves based on peak areas measured at $325 \mathrm{~nm}$, prepared for cynarin, caffeic acid, CTA, DCTA, and 5-CQA (concentration range $0.02-1.50 \mathrm{mg} \mathrm{ml}^{-1}$ ). Moreover, two caffeic acid derivatives of unknown structures being minor constituents of the analyzed extracts could be detected in some of the samples (Rt-22.04 and $24.80 \mathrm{~min}$ ). Their contents were calculated in reference to the chlorogenic acid calibration curve.

\section{Quantification of Lactucin-Like Sesquiterpene Lactones}

Sample Preparation The dried, pulverized plant material $(0.1 \mathrm{~g})$ was extracted twice with $10 \mathrm{ml}$ of $\mathrm{MeOH}$ at room temperature. The combined extracts were evaporated in vacuo and the residue was dissolved in $70 \% \mathrm{MeCN}(1 \mathrm{ml})$, left to stand overnight at $4{ }^{\circ} \mathrm{C}$, centrifuged $(11,340 \times g, 5 \mathrm{~min})$ and analyzed by RP-HPLC.

\section{RP-HPLC Separation and Quantitative Analysis}

A sample $(5 \mu \mathrm{l})$ was injected into a Purospher RP-18e $(3 \times 125 \mathrm{~mm}$, particle size $5 \mu \mathrm{m})$ column (Merck, Darmstadt, Germany) which was eluted with a mobile phase consisting of water and $\mathrm{MeCN}$, at a flow rate of $1 \mathrm{ml} \mathrm{min}^{-1}$, at $40^{\circ} \mathrm{C}$, using the aforementioned Agilent 1200 Series HPLC system. The gradient elution conditions described by Grass et al. [31] were applied. The retention time values of the analyzed compounds were as follows: $11 \beta, 13$-dihydrolactucin (1), Rt-2.7 min; lactucin (2), Rt—3.5 min; 8-deoxylactucin glucoside (crepidiaside A, 3), Rt—9.0 min; 11ß,13dihydro-8-deoxylactucin glucoside (jacquinelin glucoside, crepidiaside B, 4), Rt-10.2 min; 8deoxylactucin (5), Rt-13.5 min; 11 $\beta, 13$-dihydro-8-deoxylactucin (jacquinelin) (6), Rt-15.1 min; lactucopicrin/11ß,13-dihydrolactucopicrin (7), Rt-26.8 min (Fig. 2). Quantification was done by measurement of peak areas at $260 \mathrm{~nm}$ with a reference to the standard curve derived from four concentrations ( 0.001 to $1.000 \mathrm{mg} \mathrm{ml}^{-1}$ ) of 8-deoxylactucin glucoside.

\section{DPPH Radical Scavenging Assay}

The dried and pulverized plant material $(100 \mathrm{mg}$ ) was extracted twice with $12.5 \mathrm{ml}$ of $50 \%$ $\mathrm{MeOH}$ at room temperature. The combined extracts were evaporated in vacuo. The obtained residue was dissolved in $1 \mathrm{ml}$ of $70 \% \mathrm{MeOH}$, left to stand overnight at $4{ }^{\circ} \mathrm{C}$, centrifuged $(11,340 \times g, 5 \mathrm{~min})$ and the supernatant was diluted ten or a hundred times to obtain concentrations corresponding to $10 \mathrm{mg}$ or $1 \mathrm{mg}$ of the dry plant material per $1 \mathrm{ml}$ of the sample, respectively. DPPH (Sigma-Aldrich, USA) was dissolved in methanol to obtain a stable free radical solution $(100 \mu \mathrm{M})$. A solution $(4 \mathrm{mM})$ of 6-hydroxy-2,5,7,8tetramethylchroman-2-carboxylic acid (Trolox, reference compound) was prepared by dissolving of $100 \mathrm{mg}$ Trolox (Sigma-Aldrich, USA) in $100 \mathrm{ml}$ of methanol. To a spectrophotometric cuvette (1 cm pathlength) containing $480 \mu$ lof the methanolic DPPH solution $20 \mu \mathrm{l}$ of the diluted plant extract (final concentration $10 \mathrm{mg} \mathrm{DW} \mathrm{ml}^{-1}$ or $1 \mathrm{mg} \mathrm{DW} \mathrm{ml}^{-1}$ ) were added. A decrease in absorbance at $\lambda=517 \mathrm{~nm}$ was measured by UV/VIS CE 2021 spectrophotometer (Cecil, UK) after $1 / 2,1,2,3,5,10,15,20$, and $30 \mathrm{~min}$. DPPH radical scavenging capacity was calculated according to the following equation: $Q$ (quenching, $\%)=100\left(A_{\mathrm{o}}-A_{c}\right) / A_{\mathrm{o}}$, where $A_{\mathrm{o}}$ is 

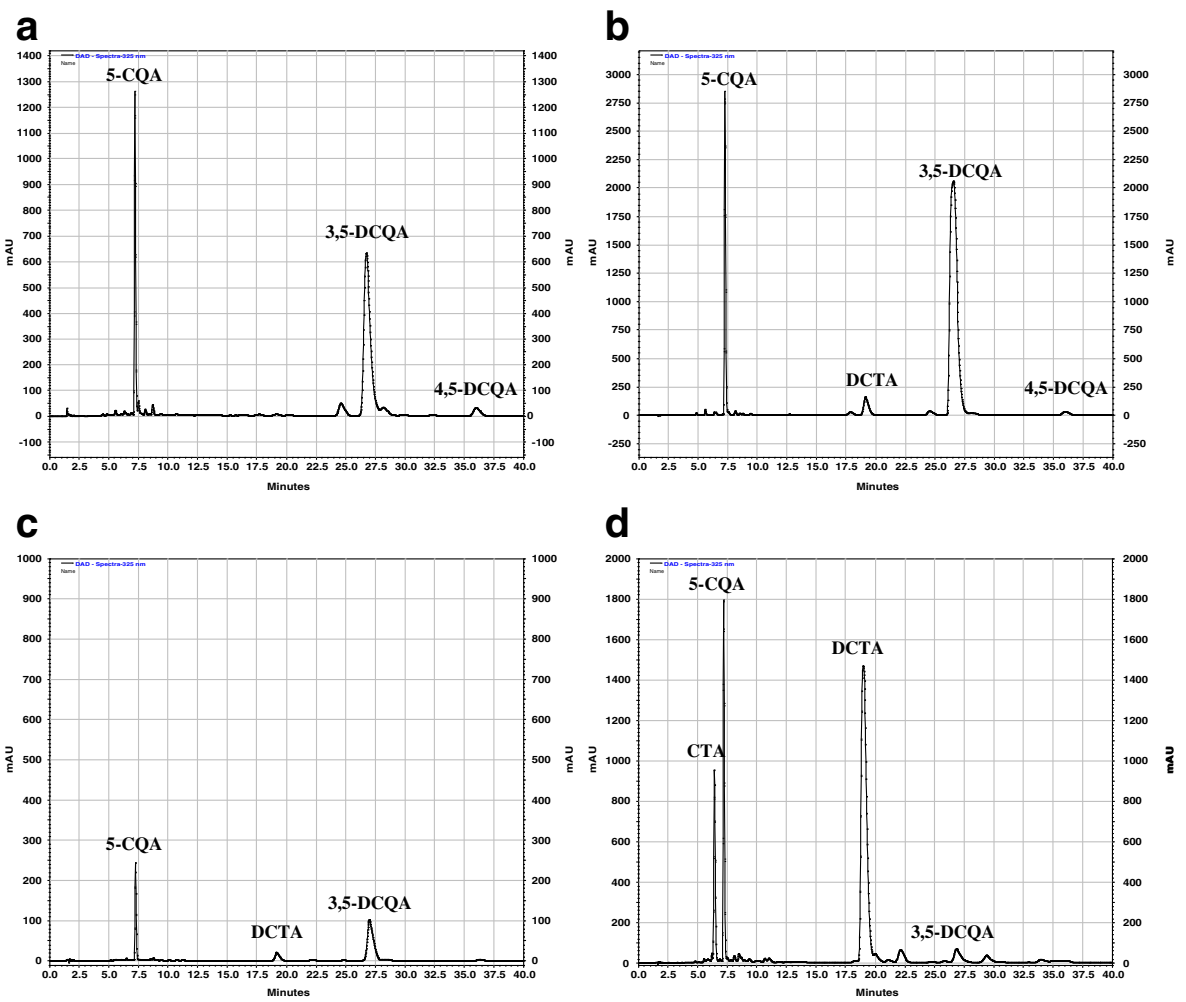

Fig. 1 RP-HPLC chromatograms of hydroxycinnamates from hydroalcoholic extracts of Cichorium intybus (detection wavelength $=325 \mathrm{~nm}$ ): a callus tissue, $\mathbf{b}$ hairy roots, $\mathbf{c}$ roots of the intact plant, $\mathbf{d}$ leaves of the intact plant. CTA caftaric acid, 5-CQA chlorogenic acid, DCTA cichoric acid, 3,5-DCQA 3,5-dicaffeoylquinic acid, 4,5-DCQA 4,5-dicaffeoylquinic acid

the absorbance of the control (in the absence of any sample) and $A_{c}$ is the value for added sample concentration $c$ [32].

\section{Estimation of Total Phenolic Content}

A reducing capacity of the plant material (total phenolic content) was estimated using FolinCiocalteu reagent as described by Velioglu et al. [33]. Results are expressed as ferulic and gallic acid equivalents.

\section{Results}

\section{Hydroxycinnamate and Caffeic Acid Contents}

Results of hydroxycinnamate quantification in the examined plant material are presented in Table 1. Contents of the compounds in hairy roots of $C$. intybus were at least one order of magnitude higher than those found in roots of the intact plant and reached their maxima at the beginning of the stationary phase of the root growth. The total content of hydroxycinnamates in the biomass from the transformed root culture was also higher $(7.0 \% \mathrm{DW})$ than that in the roots 
a

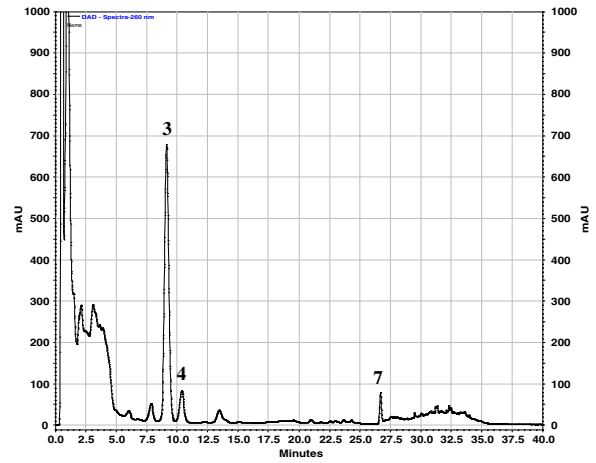

b

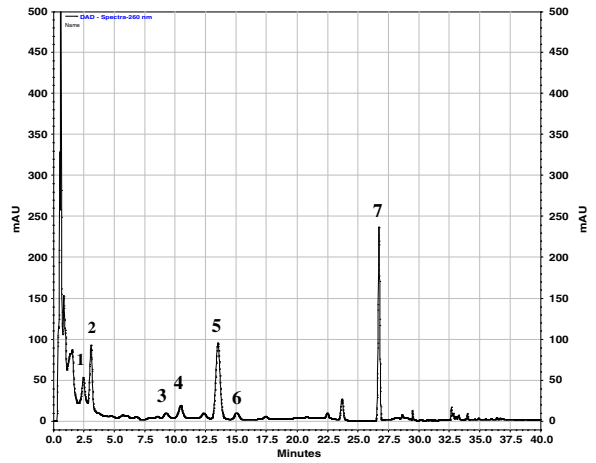

C

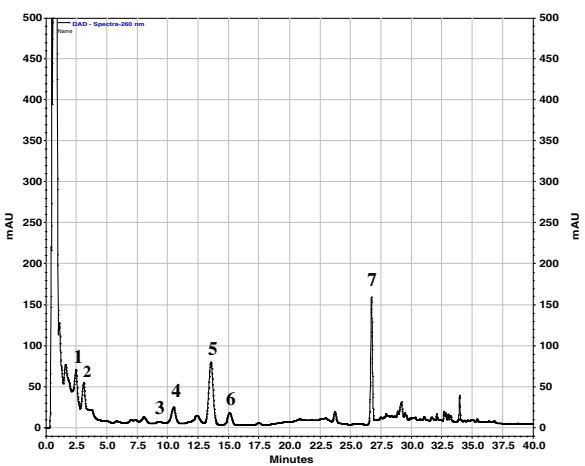

Fig. 2 RP-HPLC chromatograms of lactucin-like guaianolides from methanolic extracts of Cichorium intybus L. (detection wavelength=260 nm): a hairy roots, b roots of the intact plant, $\mathbf{c}$ leaves of the intact plant. $111 \beta, 13-$ dihydrolactucin, 2 lactucin, 3 8-deoxylactucin glucoside (crepidiaside A), 4 11 $\beta$,13-dihydro-8-deoxylactucin glucoside (crepidiaside B), 5 8-deoxylactucin, 6 11ß,13-dihydro-8-deoxylactucin (jacquinelin), 7 lactucopicrin/ $11 \beta, 13$-dihydrolactucopicrin

$(0.3-0.5 \%$ DW) and leaves (3.6 \% DW) of chicory. Moreover, substantial quantitative differences in the hydroxycinnamate patterns of the leaf and hairy root extracts were observed (Fig. 1). The transformed roots accumulated mainly 3,5-DCQA (5.5\% DW) whereas DCTA was the major phenolic constituent of the chicory leaves $(2.5 \% \mathrm{DW})$. Likewise in the hairy roots, 3,5-DCQA was the most abundant hydroxycinnamate found in the callus tissue. Unbound caffeic acid was present in the analyzed samples in minute amounts. Its content in the hairy roots of chicory reached $0.003 \pm 0.0008 \% \mathrm{DW}$ and was higher than that found in the roots of the intact plant $(0.001 \pm 0.0001 \% \mathrm{DW})$. Leaves of $C$. intybus and the callus tissue of the plant contained up to $0.01 \pm 0.0006 \%$ DW of free caffeic acid.

\section{Lactucin-Like Sesquiterpene Lactone Content}

Contents of lactucin-like guaianolides, i.e., 11ß,13-dihydrolactucin, lactucin, 8-deoxylactucin glucoside (crepidiaside A), jacquinelin glucoside (crepidiaside B), 8-deoxylactucin, jacquinelin, and lactucopicrin found in the plant material under study are shown in Table 2. The highest content of the analyzed compounds $(1.6 \% \mathrm{DW})$ was estimated in the hairy roots of $C$. intybus. The plant material contained lactucin-like guaianolides almost exclusively in their glucosylated forms (Fig. 2). 8-Deoxylactucin glucoside constituted over $85 \%$ of the sesquiterpene lactones 


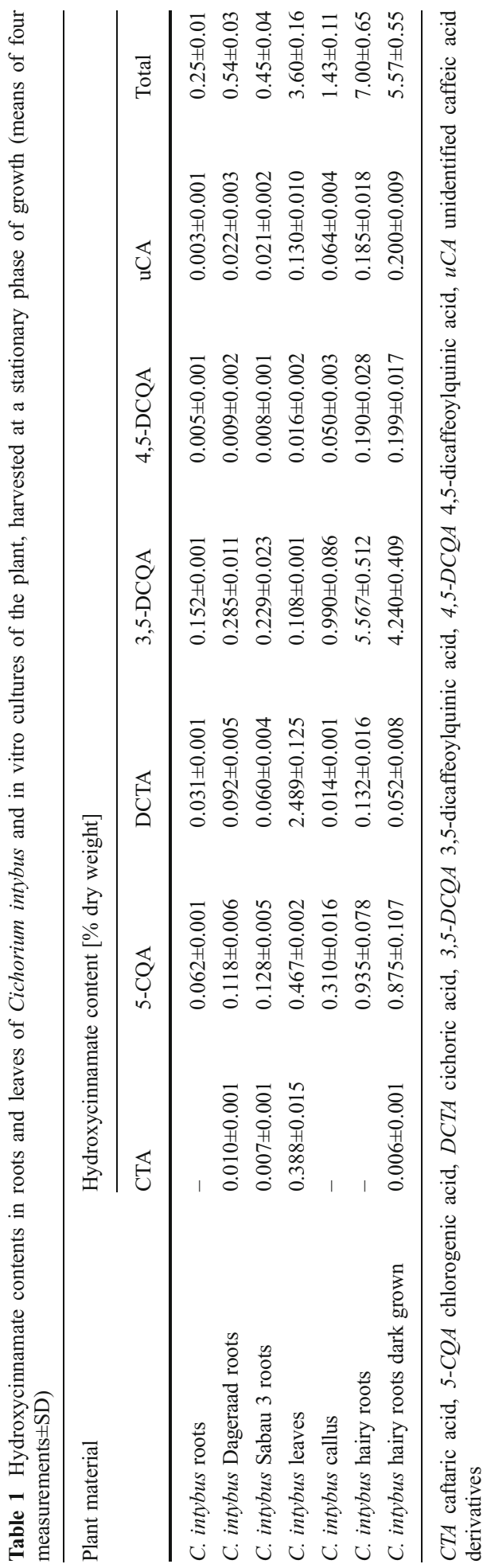




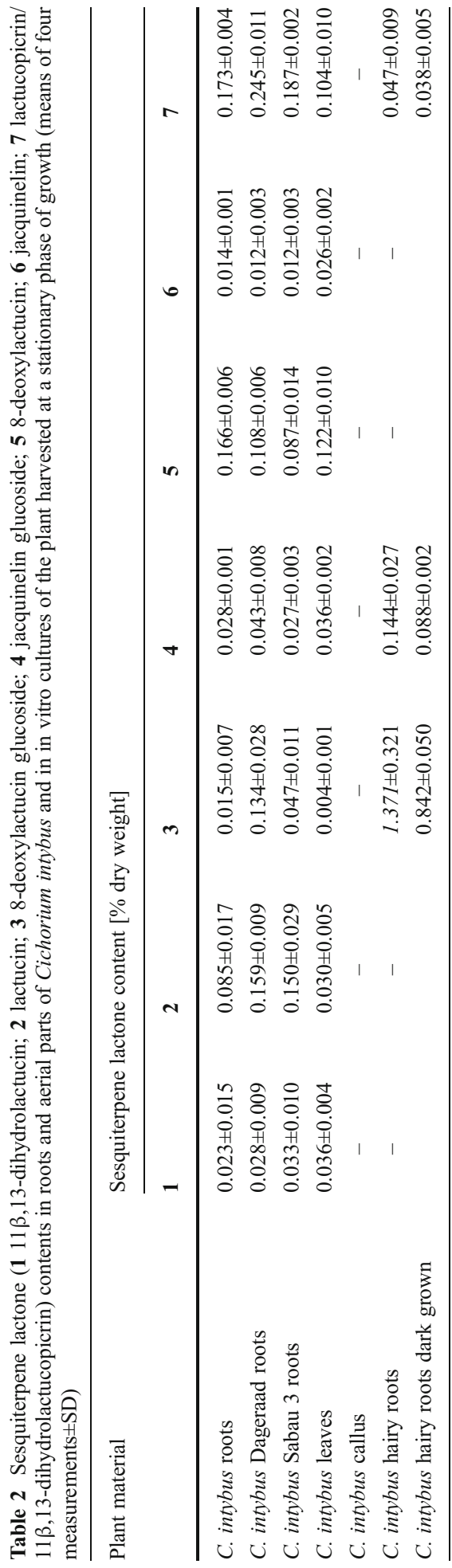


Table 3 Time course of biomass, chlorogenic acid (5-CQA), 3,5-dicaffeoylquinic acid (3,5-DCQA), 8deoxylactucin glucoside (3), jacquinelin glucoside (4), and lactucopicrin/11 $\beta, 13$-dihydrolactucopicrin (7) accumulation in hairy roots of $C$. intybus

\begin{tabular}{llllllll}
\hline $\begin{array}{l}\text { Time } \\
\text { [days }]\end{array}$ & $\begin{array}{l}\text { FW/flask } \\
{[\mathrm{g}]^{\mathrm{a}}}\end{array}$ & $\begin{array}{l}\text { DW/flask } \\
{[\mathrm{g}]^{\mathrm{a}}}\end{array}$ & \multicolumn{5}{l}{ Secondary metabolite content $[\% \mathrm{DW}]^{\mathrm{a}}$} \\
\cline { 4 - 8 } & & & 5 -CQA & 3,5 -DCQA & 3 & 4 & 7 \\
\hline 5 & $1.71 \pm 0.21$ & $0.145 \pm 0.017$ & $0.448 \pm 0.005$ & $4.201 \pm 0.145$ & $1.456 \pm 0.048$ & $0.105 \pm 0.006$ & - \\
10 & $4.02 \pm 0.72$ & $0.250 \pm 0.047$ & $0.526 \pm 0.120$ & $4.869 \pm 0.760$ & $2.502 \pm 0.702$ & $0.148 \pm 0.015$ & $0.004 \pm 0.001$ \\
15 & $6.77 \pm 0.49$ & $0.521 \pm 0.043$ & $0.788 \pm 0.118$ & $5.454 \pm 0.726$ & $1.763 \pm 0.225$ & $0.145 \pm 0.006$ & $0.029 \pm 0.005$ \\
20 & $10.44 \pm 0.39$ & $0.749 \pm 0.074$ & $0.945 \pm 0.038$ & $5.575 \pm 0.509$ & $1.554 \pm 0.238$ & $0.123 \pm 0.002$ & $0.032 \pm 0.003$ \\
28 & $11.31 \pm 0.80$ & $0.731 \pm 0.072$ & $0.948 \pm 0.107$ & $5.488 \pm 0.697$ & $1.476 \pm 0.484$ & $0.142 \pm 0.042$ & $0.051 \pm 0.001$ \\
\hline
\end{tabular}

${ }^{\mathrm{a}}$ Means of three measurements $\pm \mathrm{SD}$

$(1.4 \% \mathrm{DW})$. The time course experiment (Table 3) revealed that 10 days after inoculation into the fresh medium, the content of 8-deoxylactucin glucoside in the hairy roots reached its maximum. Lactucopicrin, which was absent from the roots in the initial phase of culture, reached its maximum content at the stationary phase of culture. Trace levels of 8-deoxylactucin (data not shown) could be detected in the cultured roots 5 and 10 days after their transfer to the fresh medium. The compound became undetectable after 15 days of the culture cycle. The leaves and roots of the wild chicory plants contained $0.4 \% \mathrm{DW}$ and $0.5 \% \mathrm{DW}$ of the investigated sesquiterpene lactones, respectively. The roots of the two commercial varieties of chicory (Dageraad and Sabau 3) accumulated similar amounts of lactucin-like guaianolides as the roots of the wild plant $(0.55-0.75 \% \mathrm{DW})$. In accordance with our previous results [28], lactucin-like guaianolides characteristic of the intact plant were not detected in the callus tissue.

\section{DPPH Radical Scavenging}

Changes in the DPPH solution absorbance over time, after addition of the tested plant extracts (10 mg DW ml ${ }^{-1}$ ) and Trolox solutions: 1.0 and $0.1 \mathrm{mg} \mathrm{ml}^{-1}$ (4.0 and $0.4 \mathrm{mM}$, respectively) are shown in Fig. 3. The hydroalcoholic extract from the hairy roots of chicory was the most active in this assay, followed by the extract from the leaves of the intact plant. Both extracts exhibited significantly higher activities than those from the normal roots and the callus tissue of the plant.

\section{Total Phenolics Content}

As expected [34], total phenolics contents in the examined plant material (see Table 3) correlated with DPPH radical scavenging activities of the corresponding extracts.

\section{Discussion}

Hydroxycinnamates, flavonoids, phenolic acids, and anthocyanins (in red varieties) are considered to be responsible for high antioxidant and antiradical potential of chicory and lettuce leaves. In the present study, the hydroalcoholic extract from the hairy roots of chicory revealed unexpectedly high activity in the DPPH scavenging assay (Fig. 3). The activity of the hairy root extract was higher than that of leaves and roots of the intact plant and C. intybus callus tissue. 
2

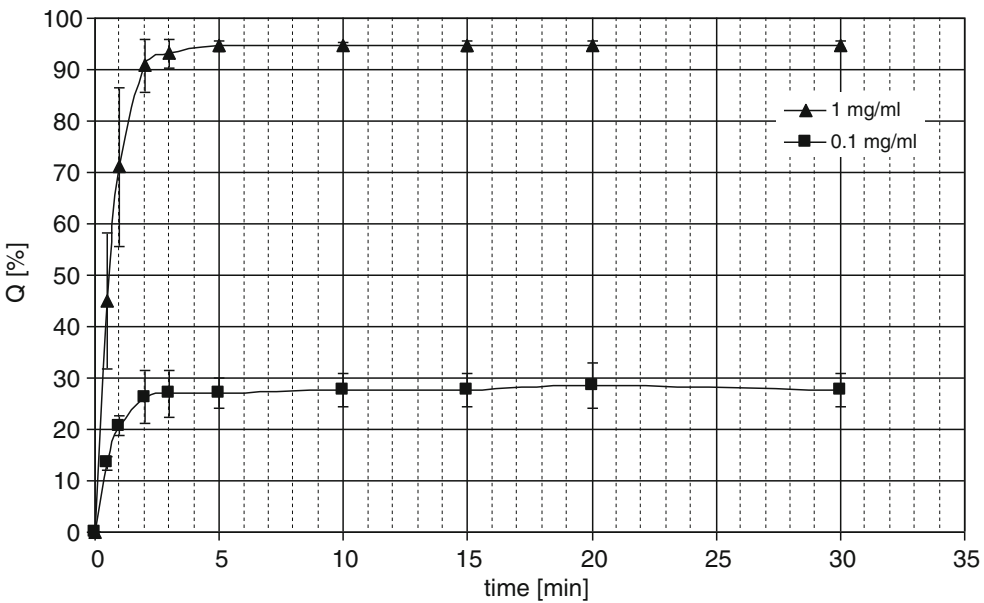

b

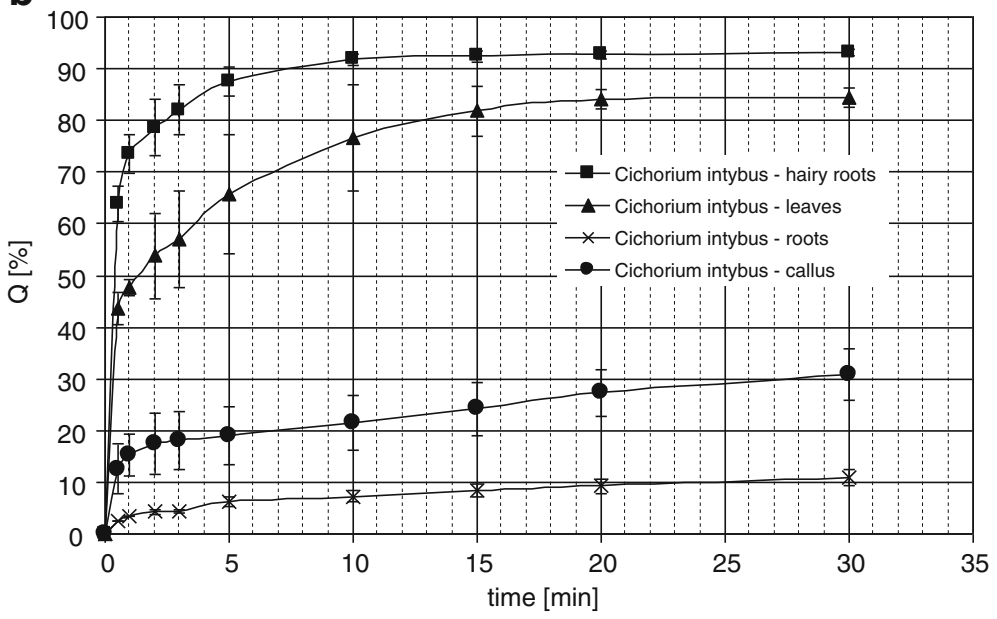

Fig. 3 DPPH radical scavenging activity of solutions of the standard compound - Trolox (a), and hydroalcoholic extracts from Cichorium intybus (concentration-10 $\mathrm{mg}$ of the dry plant material per $1 \mathrm{ml}$ of the extract) (b). $Q$ [\%] percentage of the DPPH reduction

A quantitative RP-HPLC analysis of phenolic constituents in the examined samples was performed to explain differences in their antiradical activity. Essentially similar set of signals corresponding to hydroxycinnamates was observed in every of the analyzed samples (Fig. 1). Only chromatograms of the extract from leaves of chicory showed some signals which could be ascribed to flavonoid compounds. The flavonoids (glycosides of quercetin, kaempferol, apigenin, and luteolin), which are partly responsible for antioxidant and radical scavenging activity of chicory leaves [6], were absent from both transformed roots and roots of the intact plant. A reducing capacity of the plant materials was also assessed by means of colorimetric method using Folin-Ciocalteu reagent. The total phenolic contents (Table 4) when compared with the contents of hydroxycinnamates (Table 1) clearly showed that hydroxycinnamates were responsible to high extent for antioxidant activity of the analyzed samples. According to Nicolle et al. [35], contribution of DCTA alone to the total antioxidant power of hydroalcoholic extracts 
Table 4 Reducing capacities (total phenolic contents) of extracts from roots and leaves of Cichorium intybus and in vitro cultures of the plant harvested at a stationary phase of growth. Results, expressed as ferulic acid equivalents (FA eq) and gallic acid equivalents (GA eq), are means of four measurements $( \pm \mathrm{SD})$

\begin{tabular}{lcc}
\hline \multirow{2}{*}{ Plant material } & \multicolumn{2}{c}{ Total phenolic content $[\mathrm{mg} / 100 \mathrm{~g}$ dry weight $]$} \\
\cline { 2 - 3 } & FA eq & GA eq \\
\hline C. intybus roots & $1,095 \pm 85$ & $774 \pm 39$ \\
C. intybus Dageraad roots & $1,463 \pm 208$ & $1,132 \pm 160$ \\
C. intybus Sabau 3 roots & $966 \pm 20$ & $748 \pm 15$ \\
C. intybus leaves & $5,430 \pm 51$ & $4,204 \pm 46$ \\
C. intybus callus & $3,170 \pm 71$ & $2,454 \pm 64$ \\
C. intybus hairy roots & $9,832 \pm 208$ & $7,611 \pm 161$ \\
\hline
\end{tabular}

from lettuce (L. sativa) aerial parts exceeds $50 \%$. In green varieties of chicory, DCTA was also the most efficient phenolic constituent in trapping peroxyl radicals [36]. According to our study, 3,5-DCQA was the most abundant hydroxycinnamate present in the callus and hairy root cultures of chicory. The compound also predominated in callus and hairy roots of $L$. virosa [24] and a callus culture of L. sativa var. crispa L. [37]. It is noteworthy that the hairy roots of chicory accumulated over two times more of 3,5-DCQA $(5.5 \% \mathrm{DW})$ than those of $L$. virosa $(2.6 \% \mathrm{DW})$. However, in the hairy roots of chicory, DCTA content was lower than that found in the hairy roots of lettuce. Similar differences in hydroxycinnamate pattern were observed in callus cultures of the plants. 3,5-DCQA was reported to possess hepatoprotective activity [38, 39], so it could be responsible for anti-hepatotoxic effect of a chicory callus extract described by Zafar and Ali [40]. Total content of hydroxycinnamates in the examined hairy roots of chicory was c. $20 \%$ higher $(7.0 \% \mathrm{DW})$ than that found in the chicory hairy roots grown in the dark $(5.6 \%)$ and also higher than that found in the transformed roots of $L$. virosa $(4.5 \% \mathrm{DW})$. A hairy root culture of witloof chicory (C. intybus L. cv. Lucknow local) reportedly produced coumarins, esculin and esculetin [41]. The coumarins, however, were absent from the hairy roots cultivated in our laboratory.

Lactucin-like guaianolides of chicory are often quantified after hydrolysis of their glycosidic (bounded) forms to corresponding aglycones. The procedure described by Tamaki et al. [42] is frequently adapted [43]. Very recently, another method has been reported by Ferioli and D'Antuono [44] employing a mixture of methanol, water, and formic acid as an extraction solvent. This mixture allows simultaneous extraction of sesquiterpene lactones and phenolic compounds but additional purification steps are necessary. In this study, we have observed that the presence of phenolics in the extract from the hairy roots resulted in difficulty with separation of some sesquiterpene lactones. As could be seen from Fig. 2a, hydroxycinnamates overproduced by the hairy roots are visible as a group of unseparated peaks at the initial part of the chromatogram. If lactucin and $11 \beta, 13$-dihydrolactucin were present in the extract, their quantification would be impossible. Fortunately, both compounds were absent from the hairy root biomass as shown in our previous studies [22, 23]. Figure 2 also shows that we could directly analyze both free (aglycones) and glycosidically bound sesquiterpene lactones in the methanolic extracts from the examined plant materials by RP-HPLC/DAD. The applied gradient solvent system allowed simultaneous quantification of lactucin-like guaianolides and their $11 \beta, 13$-dihydroderivatives, except for lactucopicrin and 11 $\beta, 13$-dihydrolactucopicrin which could not be resolved in the HPLC runs. The quantification of 8-deoxylactucin, described in our earlier paper [22], was done following the procedure by Peters and Amerongen [45] based on at least partial hydrolysis of sesquiterpene lactone glycosides before chromatographic analysis. The content of the compound in the transformed roots reached $0.35 \%$ calculating on a dry-weight basis and was a sum of 8 - 
deoxylactucin liberated after partial hydrolysis of its glucoside (crepidiaside A) and unbounded aglycone. In the present study, we have shown that 12-year-old hairy roots of chicory accumulated 8-deoxylactucin exclusively in its glucosylated form. In general, the transformed roots of chicory after 12 years of culture retained their capability to produce and accumulate lactucin-like guaianolides. The diversity of compounds accumulated by the hairy roots, however, remained limited in comparison to the biosynthetic potential of the intact plant [3]. The capability of roots of C. intybus intact plant to accumulate 8-deoxylactucin as the dominant sesquiterpene lactone was retained by the hairy roots of the plant, however, in the culture conditions the compound was glucosylated and stored in the cells, as no sesquiterpene lactones could be found in the culture medium. Łuczkiewicz et al. [46] reported on high content of pulchelin E in hairy roots of Rudbeckia hirta cultivated under constant light $\left(88 \pm 8 \mu \mathrm{E} \mathrm{m}^{-2} \mathrm{~s}^{-1}\right)$. The sesquiterpene lactone was absent from the intact plant and from the plant tissue cultures grown in the dark. The presence of light seems to be not a prerequisite for biosynthesis of sesquiterpene lactones in hairy roots of Cichorieae plants. Transformed roots of L. virosa, grown in the dark, produced sesquiterpene lactones characteristic of the intact plant, including lactucin-like guaianolides [24]. The hairy roots of chicory maintained in the dark also accumulated sesquiterpene lactones (Table 2). The contents of the compounds, however, were lower than those found in the roots grown under the photoperiod.

\section{Conclusions}

The biomass from A. rhizogenes-transformed root culture of chicory could be an alternative source for the production of hydroxycinnamates with well-documented antioxidative, antiradical, and hepatoprotective activities. The plant material is an exceptionally rich source of 3,5-DCQA and 5-CQA acids. Moreover, the hairy roots accumulate the sesquiterpene lactone 8deoxylactucin glycoside in high amounts. Its aglycone is known as an anti-inflammatory agent. Studies on regulatory mechanisms affecting the biosynthesis and accumulation of both phenolics and sesquiterpenes, using the investigated hairy roots as a model system, would be of interest.

Open Access This article is distributed under the terms of the Creative Commons Attribution License which permits any use, distribution, and reproduction in any medium, provided the original author(s) and the source are credited.

\section{References}

1. Baert, J. R. A. (1997). Industrial Crops and Products, 6, 195-199.

2. Amaducci, S., \& Pritoni, G. (1998). Industrial Crops and Products, 7, 345-349.

3. Kisiel, W., \& Zielińska, K. (2001). Phytochemistry, 57, 523-527.

4. Rees, S. B., \& Harborne, J. B. (1985). Phytochemistry, 24, 2225-2231.

5. Innocenti, M., Gallori, S., Giaccherini, C., Ieri, F., Vincieri, F. F., \& Mulinacci, N. (2005). Journal of Agricultural and Food Chemistry, 53, 6497-6502.

6. Heimler, D., Isolani, L., Vignolini, P., \& Romani, A. (2009). Food Chemistry, 114, 765-770.

7. Jaiswal, R., Kiprotich, J., \& Kuhnert, N. (2011). Phytochemistry, 72, 781-790.

8. Cavin, C., Delannoy, M., Malnoe, A., Debefve, E., Touche, A., Courtois, D., et al. (2005). Biochemical and Biophysical Research Communications, 327, 742-749.

9. Ripoll, C., Schmidt, B. M., Ilic, N., Poulev, A., Dey, M., Kurmukov, A. G., et al. (2007). Natural Product Communications, 2, 717-722.

10. Wesołowska, A., Nikiforuk, A., Michalska, K., Kisiel, W., \& Chojnacka-Wójcik, E. (2006). Journal of Ethnopharmacology, 107, 254-258. 
11. Ohnishi, M., Morishita, H., Iwahashi, H., Toda, S., Shirataki, Y., Kimura, M., et al. (1994). Phytochemistry, 36, 579-583.

12. Kim, H. J., \& Lee, Y. S. (2005). Planta Medica, 71, 871-876.

13. Olmos, A., Giner, R. M., Recio, M. C., Ríos, J. L., Gil-Benso, R., \& Máñez, S. (2008). Archives of Biochemistry and Biophysics, 475, 66-71.

14. Maffei Facino, R., Carini, M., Aldini, G., Saibene, L., Pietta, P., \& Mauri, P. (1995). Planta Medica, 61, $510-514$.

15. Kitagawa, S., Yoshii, K., Morita, S. Y., \& Teraoka, R. (2011). Chemical and Pharmaceutical Bulletin, 59, 793-796.

16. Robinson, W. E., Jr., Reinecke, M. G., Abdel-Malek, S., Jia, Q., \& Chow, S. A. (1996). Proceedings of the National Academy of Sciences of the United States of America, 93, 6326-6331.

17. McDougall, B., King, P. J., Wu, B. W., Hostomsky, Z., Reinecke, M. G., \& Robinson, W. E., Jr. (1998). Antimicrobial Agents and Chemotherapy, 42, 140-146.

18. Kim, K. H., Kim, Y. H., \& Lee, K. R. (2007). Bioorganic \& Medicinal Chemistry Letters, 17, 6739-6743.

19. Kodoma, M., Wada, H., Otani, H., Kohmoto, K., \& Kimura, Y. (1998). Phytochemistry, 47, $371-373$.

20. Leiss, K. A., Maltese, F., Choi, Y. H., Verpoorte, R., \& Klinkhamer, P. G. L. (2009). Plant Physiology, 150, $1567-1575$.

21. Ono, N. N., \& Tian, L. (2011). Plant Science, 180, 439-446.

22. Malarz, J., Stojakowska, A., \& Kisiel, W. (2002). Zeitschrift fur Naturforschung, 57c, 994-997.

23. Malarz, J., Stojakowska, A., Szneler, E., \& Kisiel, W. (2013). Phytochemistry Letters, 6, 59-61.

24. Stojakowska, A., Malarz, J., Szewczyk, A., \& Kisiel, W. (2012). Acta Physiologiae Plantarum, 34, $291-298$.

25. Kisiel, W., \& Gromek, D. (1993). Phytochemistry, 34, 1644-1646.

26. Kisiel, W., Barszcz, B., \& Szneler, E. (1997). Phytochemistry, 45, 365-368.

27. Kisiel, W., \& Michalska, K. (2008). Fitoterapia, 71, 86-87.

28. Malarz, J., Stojakowska, A., Szneler, E., \& Kisiel, W. (2005). Plant Cell Reports, 24, 246-249.

29. Murashige, T., \& Skoog, F. (1962). Physiologia Plantarum, 15, 473-497.

30. Spitaler, R., Schlorhaufer, P. D., Ellmerer, E. P., Merfort, I., Bortenschleger, S., Stuppner, H., et al. (2006). Phytochemistry, 67, 409-417.

31. Grass, S., Zidorn, C., Blattner, F. R., \& Stuppner, H. (2006). Phytochemistry, 67, 122-131.

32. Molyneux, P. (2004). Songklanakarin J Science and Technology, 26, 211-219.

33. Velioglu, Y. S., Mazza, G., Gao, L., \& Oomah, B. D. (1998). Journal of Agricultural and Food Chemistry, 46, 4113-4117.

34. Huang, D., Ou, B., \& Prior, R. L. (2005). Journal of Agricultural and Food Chemistry, 53, 1841-1856.

35. Nicolle, C., Carnat, A., Fraisse, D., Lamaison, J. L., Rock, E., Michel, H., et al. (2004). Journal of the Science of Food and Agriculture, 84, 2061-2069.

36. Rossetto, M., Lante, A., Vanzani, P., Spettoli, P., Scarpa, M., \& Rigo, A. (2005). Journal of Agricultural and Food Chemistry, 53, 8169-8175.

37. Tamura, H., Akioka, T., Ueno, K., Chujyo, T., Okazaki, K. I., King, P. J., et al. (2006). Molecular Nutrition \& Food Research, 50, 396-400.

38. Basnet, P., Matsushige, K., Hase, K., Kadota, S., \& Namba, T. (1996). Biological and Pharmaceutical Bulletin, 19, 1479-1484.

39. An, R. B., Sohn, D. H., Jeong, G. S., \& Kim, Y. C. (2008). Archives of Pharmacal Research, 31, 594-597.

40. Zafar, R., \& Ali, S. M. (1998). Journal of Ethnopharmacology, 63, 227-231.

41. Bais, H. P., Sudha, G., \& Ravishankar, G. A. (1999). Journal of Plant Growth Regulation, 18, $159-165$.

42. Tamaki, H., Robinson, R. W., Anderson, J. L., \& Stoewsand, G. S. (1995). Journal of Agricultural and Food Chemistry, 43, 6-8.

43. Foster, J. G., Clapham, W. M., Belesky, D. P., Labreveux, M., Hall, M. H., \& Sanderson, M. A. (2006). Journal of Agricultural and Food Chemistry, 54, 1772-1778.

44. Ferioli, F., \& D'Antuono, L. F. (2012). Food Chemistry, 135, 243-250.

45. Peters, A. M., \& van Amerongen, A. (1997). Z. Lebensmittel Untersuch Forschung A, 204, $189-193$.

46. Łuczkiewicz, M., Zárate, R., Dembińska-Migas, W., Migas, P., \& Verpoorte, R. (2002). Plant Science, $163,91-100$. 